\title{
III. Aus der Manuscriptensammlung des k. Kreisarchivs zu Amberg.
}

Von

Dr. Schneiderwirth, Reichsarchivpraktikant in München.

Das k. Kreisarchiv Amberg bewahrt unter seinen Bestanden eine Anzahl Manuscripte auf, die, wenn auch nicht gerade von hervorragender Bedeutung, so doch für die Spezialforschung immerhin manches Werthvolle aufweisen. Eine ausführliche, kritische Besprechung derselben würde an dieser Stelle nicht am Platze sein, somit begnūgen wir uns, im Folgenden einzelne Stücke, und zwar die ältesten, nach den wesentlichsten Gesichtspunkten hin zu beleuchten, vielleicht dass dadurch hier und da ein Fachmann oder Geschichtsfreund zu weiteren Studien in dieser Richtung veranlasst wird.

Zunächst sei eine "Erzehlung von dem Bayrischen Krieg und sonderlich vonder Sulzbachschen Belagerung der Behmen in Anno 1503" erwähnt; das Manuscript, ein Papierband, umfasst 21 Blätter Folio, von denen Bl. 17-21 durch Brand stark beschădigt sind, sonst ist die Schrift, der ersten Hälfte des 16. Jahrhunderts angehörend, gut leserlich. Nach einer kurzen, die Ursachen des Landshuter Erbfolgekrieges besprechenden Einleitung in Prosa, beginnt Fol. 2 die in Reimen gefasste Darstellung der Ereignisse des Jahres 1504: Mir hören von dem kaiserlichen Hoftag zu Augsburg, den Rüstungen und Bundesgenossen des Pfalzgrafen Rupreccht und Herzog Albrechts, dem allgemeinen Gang des Krieges in der Oberpfalz bis Fol. 12 der Verfasser mit den Worten: „Dabey will ichs lassen sthan, vnd von der Belagerung heben ahn" die Belagerung Sulzbachs durch die von Ruprecht geworbenen böhmischen Haufen zu erzählen beginnt. Wenn auch im allgemeinen schon bekannte Thatsachen hier überliefert werden, so verdient das Gedicht doch als von einem gleichzeitigen und 
allem Anscheine nach wahrheitsgetreuen Berichterstatter verfasst, besondere Beachtung. Dass der Erzähler Zeitgenosse und Augenzeuge, ersehen wir aus Aeusserungen, wie z. B. wo er von den Edelleuten redete, die Ruprecht beigestanden, es heisst da: 55 habe ich gezählt und 4 Ritter habe ich erkannt. Dass es ihm aber auch darum zu thun, genau und wahrhaft zu berichten, beweisen die präzisen Datenangaben für jedes bedeutende Ereigniss, sowie die wiederholten Betheuerungen, wie "das ist wahr, wie ich euch sag", „und will euch sagen, is nicht erlogen". Wer ist aber nun der Verfasser? Sein Name hat vermuthlich am Schlusse des Gedichtes gestanden, ist uns aber, weil gerade die latzten Blätter zerstört worden, nicht überliefert; ziehen wir aber Umstände in Erwägung, wie der, dass gerade die Sulabacher Belagerung, ein sonst nicht sehr bedeutsames Ereigniss des ganzen Krieges, eine ausführliche Behandlung erfährt, dass der Verfasser ferner eine besonders genaue Ortskenntniss der Umgebung genannter Stadt entwickelt, so denke ich nicht fehl zu gehen, wenn ich als den Erzähler einen Sulzbacher Bürger annehme.

Ein zweites Manuscript, 20 Bl. Folio, von denen 18 beschrieben, ebenfalls ein Papierheft, ist eine Abschrift und trägt auf der ersten Seite die Ueberschrift: "In diser Kronicken findet man zu Lesen wer Castell Stifter seien gewesen", auf der zweiten Seite: „Die Stifftung des Klosters Castel Alss Ich dass in den alten Salbücher gefunden hab Anno 1521". Es ist gleichfalls ein Gedicht, umfasst eine kurze Vorrede, in welcher die Vergänglichkeit alles irdischen Lebens besprochen wird, und 8 Capitel, deren Inhalt folgender: Von Seelandt ham ein Herzog Ernst zum Kaiser Otto II., dieser schenkte ihm den Nortwaldt in Niederbayern; Erbauung der Burg Kastel, Nachkommen Herzog Ernst's, endlich Stiftung der Abtei Kastl durch Graf Friedrich und seine Gemahlin Bertha. Dass die Darstellung, wie auch die Ueberschrift angibt, sich wenigstens in ihrer zweiten Hälfte eng an den Bericht der Salbücher anschliesst, ersehen wir u. a. aus den Worten des Verfassers, Folio 10b, „nach dem Salbuch ich das sag, dass zu Pfaffenhofen lag". Ueber den Schreiber der Chronik fohlt uns jeder Anhaltspunkt, wir ersehen nur aus dem Schluss, dass dieser am 25. Februar 1521 fertig gestellt wurde.

Akten eines vor dem Statthalter zu Zweibrücken anhängig gewesenen Ehescheidungsprozesses können wir füglich über- 
gehen, ich erwähne sie nur, weil sie uns die Erłlärung zu einigen anderen Schriftstücken geben, nämlich zu zwei Briefen Philipp Melanchthons, die mit noch einem dritten demselben Faszikel beigefügt sind. $\mathrm{Da}$ Schriften aus der Feder eines solchen Mannes stets in weiten Kreisen Aufmerksamkeit und Theilnahme orregen, mögen einige Bemerkungen über sie hier folgen. Sämmtliche drei Briefe liegen im Original vor; die zwei ersten sind in deutscher Sprache geschrieben und enthalten auf 6 resp. 5 Folioseiten Antworten auf die in erwähntem Prozesse von Belang erscheinenden Fragen. Datirt ist der letztere vom 12. November 1554. Das dritte Schreiben steht unabhängig von den vorigen $d a$, trägt einen rein privaten Charakter und ist an den herzogl. bayer. Rath Ulrich Sikinger zu Amberg gerichtet; die Sprache ist die lateinische, das Datum: „die Novembri Lutheri 1554", d. i. der 10. November.

Als ein nicht unwichtiger Beitrag zur Geschichte des Protestantismus, besonders in kirchenrechtlicher Hinsicht dürten die „Unterschiedlichen Matrimonialsachen" anzusehen sein; wir finden unter dieser Bezeichnung auf $120 \mathrm{Bl}$. Folio zunächst: „Allerhand schöne Gedanken des Wittenbergischen Consistorii in Matrimonialsachen", sodann f. 122-141 die "Constitution und Artikel des churfl. geistl. Consistorii zu Wittenberg in Sachsen anno 1542 aufgerichtet", endlich f. 142-160 die: „Lipsici Consistorii Decreta, Die Cellische Ordnung". Von beschränkter Bedeutung sind vier der Mitte des 16. Jahrhunderts angehörende lateinische Gedichte, von denen drei dem Magistrate der Stadt Nabburg, das vierte in lateinischer und griechischer Sprache dem Martin Oberdörfer, Pfarrer der Kirche zum hl. Maximilian bei Amberg gewidmet sind.

Ein weiteres hier in Betracht kommendes Manuscript führt den Titel: Dies secularis Ducatus Neuburgici. Christliche Predigt von dem ersten Seculo oder hundertjärigen zeit dess hochlöblichen Fürstenthumbs Neuburg, so vor eine. Chronik desselben kann gehalten werden. Gehalten zu Neuburg a/D. in der fürstl. Hofkirche den 30. Juli a. D. 1605. Vier Hauptpunkte werden in dieser 39 Fol. Bl. unfassenden "Predigt" besprochen: 1. Die Erhebung Neuburgs zum Fürstenthum, 2. seine erste Geschichte als solches, 3. Einführung der Reformation, 4. Anfall an das Haus Pfalz-Zweibrücken. Was den Werth des Inhaltes anbelangt, so verhält es sich damit, wie mit den meisten derartigen Quellen: vieles Bekannte wird überliefert, aber unter ihm wird der einsichtige 
Forscher inmer noch hier und da ein bisher unaufgefundenes Körnchen Wahrheit entdecken.

Von hervorragender Bedeutung insbesondere für dio Geschichte der Oberpfalz ist der hinterlassene, unter den Beständen des Archivs aufbewahrte litterarische Nachlass des verstorbenen Professors Peter Josef Moritz zu Amberg; es sind neben kleineren Arbeiten speziell diplomatischen Charakters, wie sie durch die umfangreiche Thätigkeit des Verfassers zum Besten der Monumenta Boica hier und da hervorgerufen wurden, mitunter sehr ausgedehnte Vorarbeiten über die verschiedensten Themata aus der oberpfälzischen Geschichte, wobei namentlich die Rechtsgeschichte Berücksichtigung findet. Wir geben hier einen kurzen Ueberblick:

Kritische Bemerkungen über die Stiftungsurkunde des Klosters Rott am Inn 1073. - Berichtigungen einiger histor.-genealog. Nachrichten aus der Reimchronik des Abt Hermann zu Castell ca. 1360. - Kurze Zusammenstellung der Gründe, welche beweisen können, dass die Burg, wovon sich die von 1100-1183 berühmten Grafen von Sulzbach schreiben, nur die oberpfälzische Stadt Sulzbach sein könne. - Beleuchtung eines Dokuments von 1138 des Klosters Waldsassen. - Vergleichung zweier Urkunden, welche über das oberpfälzische Foss lauten a. 1120 und a. 1212. - Bemerkungen über einen die Grafen von Sulzbach betreffenden Lehnsrevers Kaiser Friedrichs I von 1174. - Drei Sätze über eine Graffin Sophie von Hirschberg und Sulzbach. - Soweit die zusammenhängend verfassten Aufsätze, es folgen dann die noch unbearbeiteten Materialien über verschiedene Themata: M. (aterialien) über die Hofmarken und die Pfarrei Ober- und Unter-Ammerthal; 1 Convol. M. über das Pfarrdorf und die Hofmark Troschenreuth; 1 Fasz. Collektaneen zur Frage über die Mühlgilt der beiden Eckmühlen zu Türschenreuth; 1 Fasz. Vollmachtsbriefe oberpfälzischer Landsassen zu dem 1707 nach Amberg ausgeschriebenen Landtag (Copien); 1 Heft. Protokoll ïber den Landtag zu Amberg 1800; 1 Heft. Collektaneen über Ambergische Messstiftungen, nebst Grundriss der Martins-Pfarrkirche in Amberg; 1 Fasz. Excerpte aus Amberger und Sulzbacher Spitalurkunden; 1 Fasz. Materialien zu einer Geschichte der oberpfälzischen Hofmarken; 1 Fasz. Materialien zu einer historisch-geographischen Beschreibung des Landgerichts Eschenbach; 1 Fasz $M$. über Nabburg, Leuchtenberg und Waldsassen; 1 Fasz. M. zu einer Geschichte der Stadt und des Amts 
Eschenbach; 1 Fasz. 2 Aktenprodukte, betreffend eine Rocherche über das Schul- und Messnerhaus zu Wolfsbach de 1831; Plan über die Gegend zwischen Amberg und Nabburg; Correspondenz mit Pfarrer Weigl über die Geschichte von Hahnbach; 1 Fasz. Auszüge aus Herzogs Ludwig Salbuch für den Nordgau mit Bemerkungen; 1 Fasz. Ambergensia, Hohenburgensia, Hanbacensia; Urkundenauszüe von 1210, 1264-1772; 1 Conv. Ensdorfensia, ubi geographica et historica Friderici soceri Ottonis de Wittelspach, mit geographisch-antiquarischen Notizen über die Stiftungsgüter des ehemaligen Klosters Ensdorf; 1 Fasz. Excerpte aus dem Nekrolog des Schottenklosters in Regensburg und aus den Verhandlungen des Klosters Ensdorf wegen Tausches der Pfarreien Vilshofen und Püttersberg; 1 Heft. Verzeichniss oberpfälzischer Prälaten, Landsassen, Städte und Mărkte; 1 Heft. Copie oberpfälzischer Regierungserlasse wegen Landesverweisung der Juden de 1554.

Von Balth. Hiltl, churfürstl. Landes-Direktions-Sekretär zu Amberg, ist uns ein 1802 verfasster Aufsatz, $50 \mathrm{Bl}$. Fol., überliefert, der den Titel führt: Abhandlung über Oberpfälzisches Bergund Hüttenwesen in Hinsicht auf den daraus entspringenden Hauptreichthum des Landes. In eingehender Weise werden von dem Autor vornehmlich folgende Punkte erörtert: Vor Zeiten bestand der Hauptreichthum der oberen Pfalz im Berg- und Hüttenwesen, verschiedene Ursachen führten darauf einen fast allseitigen Verfall herbei, so dass zur Zeit des Verfassers dieser Industriezweig traurig darniederlag. Welche Mittel sind nun zu ergreifen, um in dieser Hinsicht einen fördernden Aufschwung zu bewirken?

Das letzte bedeutsamere Produkt, welches hier zu erwähnen, ist ein 328 halbbrüchig geschriebene Folioblätter umfassender Papierband, dessen Aufschrift: Chronicon Nordgaviense darinnen insonderheit der fürstlich pfalzgraflichen Residenzstadt Sulzbach und des vornehmen Klosters Castell Beschreibung etc. zu finden. Von einer anderen Hand ist die Bemerkung dazu geschrieben: Der Vermuthung nach vom ehemal. geheimen Referendar Grafen von Bettschart. Das Werk ist weniger Chronik als eine umfangreiche Sammlung von Auszügen, unzusammenhängend und aus mannigfachen Quellen herübergenommen, so ist namentlich die Chronik eines ehemals protestantischen Geistlichen zu Sulzbach, Namens Johann Braun mehrfach benutzt. Ein näheres Eingehen auf den reichhaltigen Inhalt würde uns hier zu weit führen, wir beschränken 
uns daher auf die Erwähnung der Hauptabschnitte. Da finden wir eine Beschreibung des Landgerichts Sulzbach, einen Bericht über die Erbauung des fürstlichen Schlosses, ein ausführliches „Verzeichniss sämntlicher Malefiz-Kosten, wie solche bis auf weitere gnädigste Verordnung ron sämmtlichen Sulzbachischen Oberämtern zu bestimmen sind", einen Aufsatz über die Grabschriften und alten Monumente im Kloster Castell etc. Umfangreich, aber von geringerem historischen Werth sind die unter der Bezeichnung „Regensburger Alterthümer" aufbewahrten handschriftlichen Materialien: Regesten, Notizen über die Regensburger Bischöfe von den ältesten Zeiten bis Albert IV. 1613-1649, Berichte über die Synoden zu Regensburg 1330 und 1465. Der Autor der Sammlung, die jedenfalls eine Vorarbeit einer Geschichte des Bisthums, ist der Pater Markus Hansizius.

Soweit unser Bericht, der nur, wie schon angedeutet, einen Theil des an angegebenem Orte aufbewahrten Materials bespricht. Sollte durch ihn hier und da ein Freund vaterländischer Geschichte zu weiteren Studien veranlasst werden, so haben wir unseren Zweck voll erreicht. 